\title{
Synthesis of Carboxyl-functionalized magnetic porous organic polymers for malachite green capture
}

\author{
LIJIN HUANG, QIN SHUAI*
}

Engineering Research Center of Nano-Geomaterials of Ministry of Education, Faculty of Materials Science and Chemistry, China University of Geosciences (Wuhan), No. 388, Lumo Road, Hongshan District, Wuhan 430074, P. R. China.

E-mail: shuaiqin@cug.edu.cn

Due to its detrimental effect even at low concentrations (e.g. $\left.0.1-10 \mathrm{mg} \mathrm{L}^{-1}\right),[1,2]$ the development of high efficiency materials for malachite green has draw worldwide attention. In this study, we reported the facile systhesis of magnetic porous organic polymers (MPOP) containing carboxylic groups and demonstrated its application in the organic contaminants removal from aquaculture wastewater. The carboxyl-rich MPOP was prepared via a facile in-situ surface polymerization strategy under mild condition. [3] And the adsorption ability of carboxyl-rich MPOP toward organic contaminants was fully studied by taking malachite green as a model. Exhilaratingly, the solution $\mathrm{pH}(\mathrm{pH} 2-12)$ had no significant impact on the adsorption and the target contaminant can be captured onto carboxyl-rich MPOP within 2 min when the initial concentration was $100.0 \mathrm{mg} \mathrm{L}^{-1}$. Furthermore, the resultant composites also exhibited very high adsorption capacities (569-779 $\mathrm{mg} \mathrm{g}^{-1}$ ). It is noteworthy that the content of carboxylic groups was positively correlated with the capacities $\left(\mathrm{R}^{2}=0.958\right)$. These results reveal that the carboxylic groups play a crucial role for the capture of malachite green. What's more, the carboxyl-rich MPOP also can be recovered easily by using a magnet and reused for 5 times without performance decrement. The resultant composite can also remove the contaminant from practical aquaculture wastewater with highly efficiency, demonstrating its high application potential for environmental remediation.

\section{References}

[1] N.A. Gavrilenko, T.N. Volgina, E.V. Pugachev, M.A. Gavrilenko, Visual determination of malachite green in sea fish samples, Food Chem 2019; 274: 242-245.

[2] K. Gupta, O.P. Khatri, Reduced graphene oxide as an effective adsorbent for removal of malachite green dye: Plausible adsorption pathways, J Colloid Interface Sci 2017; 501: 11-21.

[3] L.J. Huang, Q. Shuai, Facile approach to prepare sulfur-functionalized magnetic amide-linked organic polymers for enhanced $\mathrm{Hg}(\mathrm{II})$ removal from water, ACS Sustain Chem Eng 2019; 7: 9957-9965. 